\title{
Some new characterizations of the Bloch space
}

\author{
Songxiao $\mathrm{Li}^{1}$ and Stevo Stević $2,3^{*}$
}

"Correspondence: sstevic@ptt.rs

${ }^{2}$ Mathematical Institute of the

Serbian Academy of Sciences, Knez

Mihailova 36/III, Beograd, 11000,

Serbia

${ }^{3}$ Department of Mathematics, King

Abdulaziz University, P.O. Box 80203

Jeddah, 21589, Saudi Arabia

Full list of author information is

available at the end of the article

\section{Abstract}

We obtain some new characterizations for the Bloch space on the open unit disk in the complex plane $\mathbb{C}$ and the open unit ball of $\mathbb{C}^{n}$.

MSC: Primary 32A18

Keywords: Bloch space; unit disk; unit ball

\section{Introduction}

Let $\mathbb{D}$ be the open unit disk in the complex plane $\mathbb{C}, \mathbb{B}$ the open unit ball of the complex vector space $\mathbb{C}^{n}, H(\mathbb{D})$ the class of all holomorphic functions on $\mathbb{D}$ and $H(\mathbb{B})$ the class of all holomorphic functions on $\mathbb{B}$.

For $f \in C^{1}(\mathbb{B})$, the invariant gradient $\widetilde{\nabla} f$ is defined by

$$
(\widetilde{\nabla} f)(z)=\nabla\left(f \circ \varphi_{z}\right)(0),
$$

where

$$
\nabla f(z)=\left(\frac{\partial f}{\partial z_{1}}(z), \ldots, \frac{\partial f}{\partial z_{n}}(z)\right)
$$

is the complex gradient of $f$.

A holomorphic function $f$ in $\mathbb{D}$ is said to belong to the Bloch space $\mathcal{B}(\mathbb{D})$ if

$$
\|f\|_{\mathcal{B}}=|f(0)|+\sup _{z \in \mathbb{D}}\left(1-|z|^{2}\right)\left|f^{\prime}(z)\right|<\infty .
$$

Under the above norm, $\mathcal{B}(\mathbb{D})$ is a Banach space (see, e.g. [1]). On the unit ball, the Bloch space $\mathcal{B}(\mathbb{B})$, which was introduced by Hahn in [2], is the space of all $f \in H(\mathbb{B})$ such that

$$
\|f\|_{\mathcal{B}}=\sup _{z \in \mathbb{B}} \sup _{w \in \mathbb{C}^{n} \backslash\{0\}} \frac{|\langle\nabla f(z), \bar{w}\rangle|}{\sqrt{\frac{n+1}{2} \frac{\left(1-|z|^{2}\right)|w|^{2}+|\langle w, z\rangle|^{2}}{\left(1-|z|^{2}\right)^{2}}}}<\infty .
$$

For some classical results on Bloch spaces see [3] and [4].

It is well known that $f \in \mathcal{B}(\mathbb{B})$ if and only if (see, e.g. [5])

$$
\sup _{z \in \mathbb{B}}\left(1-|z|^{2}\right)|\nabla f(z)|<\infty .
$$

C2014 Li and Stević; licensee Springer. This is an Open Access article distributed under the terms of the Creative Commons Attribution License (http://creativecommons.org/licenses/by/2.0), which permits unrestricted use, distribution, and reproduction in any medium, provided the original work is properly cited. 
For $\alpha>0$, an $f \in H(\mathbb{B})$ is said to belong to the $\alpha$-Bloch space, denoted by $\mathcal{B}^{\alpha}(\mathbb{B})$, if

$$
\sup _{z \in \mathbb{B}}\left(1-|z|^{2}\right)^{\alpha}|\nabla f(z)|<\infty
$$

When $\alpha=1$, the $\alpha$-Bloch space is the classical Bloch space.

It is of some importance to give new characterizations for a function space, since, for example, it can be useful in the study of operators acting on the space. For example, by using the last expression, it is difficult to study composition operators on $\alpha$-Bloch space. However, in [6] Zhang and $\mathrm{Xu}$ introduced the metric

$$
F_{z}^{\alpha}(w)=\sqrt{\frac{n+1}{2}} \frac{\sqrt{\lambda_{\alpha}(|z|)|w|^{2}+\left(1-\lambda_{\alpha}(|z|)\right)|\langle w, z\rangle|^{2} /|z|^{2}}}{\left(1-|z|^{2}\right)^{\alpha}}
$$

and proved that $f \in \mathcal{B}^{\alpha}(\mathbb{B})$ if and only if

$$
\sup _{z, w \in \mathbb{C}^{n} \backslash\{0\}} \frac{|\nabla f(z) w|}{F_{z}^{\alpha}(w)}<\infty,
$$

and by using (1), they completely characterized the boundedness and compactness of composition operators on $\alpha$-Bloch spaces. For some other results on operators on Bloch-type spaces see, for example, [7-21] and the references therein.

For $f \in H(\mathbb{B})$, in $[22]$ was proved that $f \in \mathcal{B}(\mathbb{B})$ if and only if

$$
M_{1}=\sup _{\substack{z, w \in \mathbb{B} \\ z \neq w}}\left(1-|z|^{2}\right)^{1 / 2}\left(1-|w|^{2}\right)^{1 / 2} \cdot \frac{|f(z)-f(w)|}{\left|w-P_{w} z-s_{w} Q_{w} z\right|}<\infty .
$$

Somewhat later, in [23] it was proved that $f \in \mathcal{B}(\mathbb{B})$ if and only if

$$
M_{2}=\sup _{\substack{z, w \in \mathbb{B} \\ z \neq w}}\left(1-|z|^{2}\right)^{1 / 2}\left(1-|w|^{2}\right)^{1 / 2} \cdot \frac{|f(z)-f(w)|}{|z-w|}<\infty,
$$

while in [24] it was proved that $f \in \mathcal{B}(\mathbb{B})$ if and only if

$$
M_{3}=\sup _{z, w \in \mathbb{B}}\left(1-|z|^{2}\right)^{1 / 2}\left(1-|w|^{2}\right)^{1 / 2} \cdot \frac{|f(z)-f(w)|}{|1-\langle z, w\rangle|}<\infty .
$$

These characterizations can be seen as derivative-free characterizations of the Bloch space on the unit ball. For the case of the unit polydisk, see [25] and [26]. For more characterizations of Bloch-type spaces in the unit disk, unit polydisk and unit ball, see, for example, [5, 22-24, 27-49].

In this paper, we give some new characterizations for the Bloch space. In Section 2, we give some preliminary results which are used in the proofs of our main results. In Section 3, we give two new characterizations for the Bloch space on $\mathbb{D}$. In Section 4 , we give four new characterizations for the Bloch space in the unit ball $\mathbb{B}$, which, among others, generalize the corollaries in Section 3.

Throughout this paper, constants are denoted by $C$, they are positive and may differ from one occurrence to the next. We say that two quantities $K_{1}(x)$ and $K_{2}(x)$ are comparable, if 
there are positive constants $C_{1}$ and $C_{2}$ independent of variable $x$ such that

$$
C_{1} K_{1}(x) \leq K_{2}(x) \leq C_{2} K_{1}(x)
$$

\section{Preliminaries and auxiliary results}

Let $z=\left(z_{1}, \ldots, z_{n}\right)$ and $w=\left(w_{1}, \ldots, w_{n}\right)$ be points in the complex vector space $\mathbb{C}^{n}$ and $\langle z, w\rangle=$ $z_{1} \bar{w}_{1}+\cdots+z_{n} \bar{w}_{n}$. Let $\operatorname{Aut}(\mathbb{B})$ be the group of all biholomorphic selfmaps of $\mathbb{B}$. It is well known that $\operatorname{Aut}(\mathbb{B})$ is generated by the unitary operators on $\mathbb{C}^{n}$ and the involutions $\varphi_{a}$ of the form

$$
\varphi_{a}(z)=\frac{a-P_{a} z-s_{a} Q_{a} z}{1-\langle z, a\rangle},
$$

where $s_{a}=\left(1-|a|^{2}\right)^{1 / 2}, P_{a}$ is the orthogonal projection into the space spanned by $a \in \mathbb{B}$, i.e.,

$$
P_{a} z=\frac{\langle z, a\rangle a}{|a|^{2}}, \quad|a|^{2}=\langle a, a\rangle, \quad P_{0} z=0
$$

and $Q_{a}=I-P_{a}$. See $[5,50]$ for more properties of $\varphi_{a}(z)$.

Recall that the weighted Bergman space $A_{\alpha}^{p}(\mathbb{B})$, where $0<p<\infty$ and $\alpha>-1$, consists of those functions $f \in H(\mathbb{B})$ for which

$$
\|f\|_{A_{\alpha}^{p}}^{p}=\int_{\mathbb{B}}|f(z)|^{p} d v_{\alpha}(z)=c_{\alpha} \int_{\mathbb{B}}|f(z)|^{p}\left(1-|z|^{2}\right)^{\alpha} d v(z)<\infty,
$$

where $c_{\alpha}=\frac{\Gamma(n+\alpha+1)}{n ! \Gamma(\alpha+1)}, d v$ is the normalized Lebesgue measure of $\mathbb{B}($ i.e. $v(\mathbb{B})=1)$. When $n=1$, we denote $d v_{\alpha}$ by $d A_{\alpha}$. When $\alpha=0$, we get the classical Bergman space, which will be denoted by $A^{p}=A^{p}(\mathbb{B})$.

Let

$$
d \lambda(z)=\frac{d v(z)}{\left(1-|z|^{2}\right)^{n+1}} .
$$

For any $\psi \in A u t(\mathbb{B})$ and $f \in L^{1}(\mathbb{B})$,

$$
\int_{\mathbb{B}} f(z) d \lambda(z)=\int_{\mathbb{B}} f \circ \psi(z) d \lambda(z)
$$

Thus $d \lambda(z)$ is a Möbius invariant measure (see, e.g. [36]).

Next, we quote some well-known results that will be used in the proofs of our main results. We begin with the following characterization of the Bloch space in the unit ball (see [5, 32, 34]).

Lemma 2.1 Let $0<p<\infty$. A holomorphic function $f$ is in the Bloch space $\mathcal{B}(\mathbb{B})$ if and only if

$$
\sup _{a \in \mathbb{B}}\left\|f \circ \varphi_{a}-f(a)\right\|_{A^{p}}<\infty
$$


In [51], are proved the following two characterizations for the weighted Bergman space in the unit ball.

Lemma 2.2 Assume that $0<p<\infty, \alpha>-1$ and $f \in H(\mathbb{B})$. If $\beta$ and $\gamma$ are real parameters such that

$$
\beta+\gamma=\alpha+p-(n+1)
$$

and

$$
-1<\beta<p-(n+1), \quad-1<\gamma<p-(n+1),
$$

then the following statements are equivalent:

(a) $f \in A_{\alpha}^{p}(\mathbb{B})$;

(b)

$$
Q_{1}(f):=\int_{\mathbb{B}} \int_{\mathbb{B}} \frac{|f(z)-f(w)|^{p}}{|z-w|^{p}} d v_{\beta}(z) d v_{\gamma}(w)<\infty
$$

(c)

$$
Q_{2}(f):=\int_{\mathbb{B}} \int_{\mathbb{B}} \frac{|f(z)-f(w)|^{p}}{|1-\langle z, w\rangle|^{p}} d v_{\beta}(z) d v_{\gamma}(w)<\infty
$$

Moreover, the quantities $Q_{1}(f), Q_{2}(f)$, and $\|f\|_{A_{\alpha}^{p}}^{p}$ are comparable.

Lemma 2.3 [32] Assume that $f \in H(\mathbb{B}), 0<p<\infty,-1<q<\infty, 0 \leq s<\infty, 0 \leq t<p+2 n$, and $p+s>n$. Then for $a \in \mathbb{B}$,

$$
\begin{aligned}
& \int_{\mathbb{B}} \frac{|f(z)-f(0)|^{p}}{|z|^{t}}\left(1-|z|^{2}\right)^{q}\left(1-\left|\varphi_{a}(z)\right|^{2}\right)^{s} d v(z) \\
& \quad \leq C \int_{\mathbb{B}}|\widetilde{\nabla} f|^{p}\left(1-|z|^{2}\right)^{q}\left(1-\left|\varphi_{a}(z)\right|^{2}\right)^{s} d v(z) .
\end{aligned}
$$

Lemma 2.4 [32] Assume that $f \in H(\mathbb{B})$ and $0<p<\infty$. Then $f \in \mathcal{B}(\mathbb{B})$ if and only if

$$
\sup _{a \in \mathbb{B}} \int_{\mathbb{B}}|\widetilde{\nabla} f(z)|^{p}\left(1-\left|\varphi_{a}(z)\right|^{2}\right)^{n+1} d \lambda(z)<\infty .
$$

The following well-known result can be found in [50] or [5].

Lemma 2.5 Let $-1<t<\infty$ and $c \in \mathbb{R}$. Then there is a positive constant $C$ such that

$$
\int_{\mathbb{B}} \frac{\left(1-|z|^{2}\right)^{t}}{|1-\langle z, w\rangle|^{n+1+t+c}} d v(z) \begin{cases}\leq \frac{C}{\left(1-|w|^{2} c^{c}\right.}, & \text { if } c>0, \\ \leq C \log \frac{e}{1-|w|^{2}}, & \text { if } c=0, \\ \text { is bounded, } & \text { if } c<0,\end{cases}
$$

for all $w \in \mathbb{B}$. 


\section{Characterizations of the Bloch space in the unit disk}

In this section, we give two characterizations for the Bloch space in the unit disk as follows.

Theorem 3.1 Assume that $f \in H(\mathbb{D})$ and $0<p<\infty$. If $\beta$ and $\gamma$ are real parameters satisfying the following conditions:

$$
\beta+\gamma=p-2, \quad-1<\beta<p-2, \quad-1<\gamma<p-2,
$$

then the following statements are equivalent:

(a) $f \in \mathcal{B}(\mathbb{D})$;

(b)

$$
\sup _{a \in \mathbb{D}} \int_{\mathbb{D}} \int_{\mathbb{D}} \frac{|f(z)-f(w)|^{p}}{|z-w|^{p}} \frac{\left(1-|a|^{2}\right)^{2}\left(1-|z|^{2}\right)^{\beta}\left(1-|w|^{2}\right)^{\gamma}}{|1-\bar{a} z|^{\beta-\gamma+2}|1-\bar{a} w|^{\gamma-\beta+2}} d A(z) d A(w)<\infty ;
$$

(c)

$$
\sup _{a \in \mathbb{D}} \int_{\mathbb{D}} \int_{\mathbb{D}} \frac{|f(z)-f(w)|^{p}}{|1-\bar{z} w|^{p}} \frac{\left(1-|a|^{2}\right)^{2}\left(1-|z|^{2}\right)^{\beta}\left(1-|w|^{2}\right)^{\gamma}}{|1-\bar{a} z|^{\beta-\gamma+2}|1-\bar{a} w|^{\gamma-\beta+2}} d A(z) d A(w)<\infty .
$$

Proof By taking $n=1, \alpha=0$ in Lemma 2.2, we see that $f \in A^{p}(\mathbb{D})$ if and only if

$$
\int_{\mathbb{D}} \int_{\mathbb{D}} \frac{|f(z)-f(w)|^{p}}{|z-w|^{p}}\left(1-|z|^{2}\right)^{\beta}\left(1-|w|^{2}\right)^{\gamma} d A(z) d A(w)<\infty
$$

and if and only if

$$
\int_{\mathbb{D}} \int_{\mathbb{D}} \frac{|f(z)-f(w)|^{p}}{|1-\bar{z} w|^{p}}\left(1-|z|^{2}\right)^{\beta}\left(1-|w|^{2}\right)^{\gamma} d A(z) d A(w)<\infty
$$

when the conditions in (11) hold.

Replacing $f$ by $f \circ \varphi_{a}-f(a)$ in (12) and (13), and using Lemma 2.1, we conclude that $f \in \mathcal{B}(\mathbb{D})$ if and only if

$$
\sup _{a \in \mathbb{D}} \int_{\mathbb{D}} \int_{\mathbb{D}} \frac{\left|f \circ \varphi_{a}(z)-f \circ \varphi_{a}(w)\right|^{p}}{|z-w|^{p}}\left(1-|z|^{2}\right)^{\beta}\left(1-|w|^{2}\right)^{\gamma} d A(z) d A(w)<\infty,
$$

which is equivalent to

$$
\sup _{a \in \mathbb{D}} \int_{\mathbb{D}} \int_{\mathbb{D}} \frac{\left|f \circ \varphi_{a}(z)-f \circ \varphi_{a}(w)\right|^{p}}{|1-\bar{z} w|^{p}}\left(1-|z|^{2}\right)^{\beta}\left(1-|w|^{2}\right)^{\gamma} d A(z) d A(w)<\infty .
$$

Using the change of variables $z \mapsto \varphi_{a}(z), w \mapsto \varphi_{a}(w)$ and the following equalities (see, e.g. [1]):

$$
\left|\varphi_{a}(z)-\varphi_{a}(w)\right|=\frac{|z-w|\left(1-|a|^{2}\right)}{|1-\bar{a} w||1-\bar{a} z|}
$$

and

$$
\left|1-\overline{\varphi_{a}(z)} \varphi_{a}(w)\right|=\frac{|1-\bar{z} w|\left(1-|a|^{2}\right)}{|1-\bar{a} w||1-\bar{a} z|},
$$


we see that the double integrals on the left side of (14) and (15) are equivalent to

$$
\sup _{a \in \mathbb{D}} \int_{\mathbb{D}} \int_{\mathbb{D}} \frac{|f(z)-f(w)|^{p}}{|z-w|^{p}} \frac{\left(1-|a|^{2}\right)^{2}\left(1-|z|^{2}\right)^{\beta}\left(1-|w|^{2}\right)^{\gamma}}{|1-\bar{a} z|^{\beta-\gamma+2}|1-\bar{a} w|^{\gamma-\beta+2}} d A(z) d A(w)
$$

and

$$
\sup _{a \in \mathbb{D}} \int_{\mathbb{D}} \int_{\mathbb{D}} \frac{|f(z)-f(w)|^{p}}{|1-\bar{z} w|^{p}} \frac{\left(1-|a|^{2}\right)^{2}\left(1-|z|^{2}\right)^{\beta}\left(1-|w|^{2}\right)^{\gamma}}{|1-\bar{a} z|^{\beta-\gamma+2}|1-\bar{a} w|^{\gamma-\beta+2}} d A(z) d A(w),
$$

respectively. Therefore, $f \in \mathcal{B}(\mathbb{D})$ if and only if (b) holds, and if and only if (c) holds, as desired.

Taking $\beta=\gamma=p / 2-1$ in Theorem 3.1, we easily get the following corollary.

Corollary 3.1 Assume that $f \in H(\mathbb{D})$ and $2<p<\infty$. Then the following statements are equivalent:

(a) $f \in \mathcal{B}(\mathbb{D})$;

(b)

$$
\sup _{a \in \mathbb{D}} \int_{\mathbb{D}} \int_{\mathbb{D}}\left(\frac{|f(z)-f(w)|}{|z-w|}\right)^{p}\left(1-\left|\varphi_{a}(z)\right|^{2}\right)\left(1-\left|\varphi_{a}(w)\right|^{2}\right) d A_{t}(z) d A_{t}(w)<\infty ;
$$

(c)

$$
\sup _{a \in \mathbb{D}} \int_{\mathbb{D}} \int_{\mathbb{D}}\left(\frac{|f(z)-f(w)|}{|1-\bar{z} w|}\right)^{p}\left(1-\left|\varphi_{a}(z)\right|^{2}\right)\left(1-\left|\varphi_{a}(w)\right|^{2}\right) d A_{t}(z) d A_{t}(w)<\infty
$$

where $t=(p-4) / 2$.

Taking $\beta=p / 2-2, \gamma=p / 2$ in Theorem 3.1, we can easily get the following result.

Corollary 3.2 Assume that $f \in H(\mathbb{D})$ and $4<p<\infty$. Then the following statements are equivalent:

(a) $f \in \mathcal{B}(\mathbb{D})$;

(b)

$$
\sup _{a \in \mathbb{D}} \int_{\mathbb{D}} \int_{\mathbb{D}}\left(\frac{|f(z)-f(w)|}{|z-w|}\right)^{p}\left(1-\left|\varphi_{a}(w)\right|^{2}\right)^{2} d A_{t}(z) d A_{t}(w)<\infty ;
$$

(c)

$$
\sup _{a \in \mathbb{D}} \int_{\mathbb{D}} \int_{\mathbb{D}}\left(\frac{|f(z)-f(w)|}{|1-\bar{z} w|}\right)^{p}\left(1-\left|\varphi_{a}(w)\right|^{2}\right)^{2} d A_{t}(z) d A_{t}(w)<\infty,
$$

where $t=(p-4) / 2$.

\section{Characterizations of the Bloch space in the unit ball}

In this section, we generalize Corollaries 3.1 and 3.2 in the setting of the unit ball. 
Theorem 4.1 Assume that $f \in H(\mathbb{B})$ and $n+1<p<\infty$. Then $f \in \mathcal{B}(\mathbb{B})$ if and only if

$$
\sup _{a \in \mathbb{B}} \int_{\mathbb{B}} \int_{\mathbb{B}}\left(\frac{|f(z)-f(w)|}{|1-\langle z, w\rangle|}\right)^{p}\left(1-\left|\varphi_{a}(z)\right|^{2}\right)^{\frac{n+1}{2}}\left(1-\left|\varphi_{a}(w)\right|^{2}\right)^{\frac{n+1}{2}} d v_{t}(z) d v_{t}(w)<\infty,
$$

where $t=(p-2(n+1)) / 2$.

Proof Let $\alpha=0$ and $\beta=\gamma=(p-(n+1)) / 2$ in Lemma 2.2. We see that $f \in A_{\alpha}^{p}(\mathbb{B})$ if and only if

$$
\int_{\mathbb{B}} \int_{\mathbb{B}}\left(\frac{|f(z)-f(w)|}{|1-\langle z, w\rangle|}\left(1-|z|^{2}\right)^{1 / 2}\left(1-|w|^{2}\right)^{1 / 2}\right)^{p} d v_{k}(z) d v_{k}(w)<\infty
$$

where $k=-(n+1) / 2$.

From this and by Lemma 2.1 we see that $f \in \mathcal{B}(\mathbb{B})$ if and only if

$$
\sup _{a \in \mathbb{B}} \int_{\mathbb{B}} \int_{\mathbb{B}}\left(\frac{\left|f \circ \varphi_{a}(z)-f \circ \varphi_{a}(w)\right|}{|1-\langle z, w\rangle|}\left(1-|z|^{2}\right)^{\frac{1}{2}}\left(1-|w|^{2}\right)^{\frac{1}{2}}\right)^{p} d v_{k}(z) d v_{k}(w)<\infty .
$$

Using the change of variables $z \mapsto \varphi_{a}(z), w \mapsto \varphi_{a}(w)$ and (4), we see that the left side of the last inequality is equivalent to

$$
\sup _{a \in \mathbb{B}} \int_{\mathbb{B}} \int_{\mathbb{B}} \frac{|f(z)-f(w)|^{p}\left(1-\left|\varphi_{a}(z)\right|^{2}\right)^{\frac{p+n+1}{2}}\left(1-\left|\varphi_{a}(w)\right|^{2}\right)^{\frac{p+n+1}{2}}}{\left|1-\left\langle\varphi_{a}(z), \varphi_{a}(w)\right\rangle\right|^{p}} d \lambda(z) d \lambda(w) .
$$

The result follows by using the equalities (see, e.g. [5])

$$
1-\left\langle\varphi_{a}(z), \varphi_{a}(w)\right\rangle=\frac{(1-\langle a, a\rangle)(1-\langle z, w\rangle)}{(1-\langle z, a\rangle)(1-\langle a, w\rangle)}
$$

and

$$
1-\left|\varphi_{a}(z)\right|^{2}=\frac{\left(1-|a|^{2}\right)\left(1-|z|^{2}\right)}{|1-\langle z, a\rangle|^{2}}
$$

in (16).

Theorem 4.2 Assume that $f \in H(\mathbb{B})$ and $2 n<p<\infty$. Then $f \in \mathcal{B}(\mathbb{B})$ if and only if

$$
\sup _{a \in \mathbb{B}} \int_{\mathbb{B}} \int_{\mathbb{B}}\left(\frac{|f(z)-f(w)|}{\left|w-P_{w} z-s_{w} Q_{w} z\right|}\right)^{p}\left(1-\left|\varphi_{a}(z)\right|^{2}\right)^{\frac{n+1}{2}}\left(1-\left|\varphi_{a}(w)\right|^{2}\right)^{\frac{n+1}{2}} d v_{t}(z) d v_{t}(w)<\infty,
$$

where $t=(p-2(n+1)) / 2$.

Proof Suppose that (17) holds. Since

$$
\frac{1}{|1-\langle z, w\rangle|} \leq \frac{1}{\left|w-P_{w} z-s_{w} Q_{w} z\right|}, \quad z, w \in \mathbb{B} .
$$

Then by Theorem 4.1 and (18) we see that $f \in \mathcal{B}(\mathbb{B})$. 
Conversely, suppose that $f \in \mathcal{B}(\mathbb{B})$. By using the change of variables $z \rightarrow \varphi_{w}(u)$, Lemma 2.3 and the following equality (see [5]):

$$
\frac{1}{1-\left\langle\varphi_{w}(u), w\right\rangle}=\frac{1-\langle u, w\rangle}{1-|w|^{2}}, \quad u, w \in \mathbb{B}
$$

we have

$$
\begin{aligned}
& \int_{\mathbb{B}} \int_{\mathbb{B}} \frac{|f(z)-f(w)|^{p}\left(1-\left|\varphi_{a}(z)\right|^{2}\right)^{\frac{n+1}{2}}\left(1-\left|\varphi_{a}(w)\right|^{2}\right)^{\frac{n+1}{2}}}{\left|w-P_{w} z-s_{w} Q_{w} z\right|^{p}} d v_{t}(z) d v_{t}(w) \\
& \quad=\int_{\mathbb{B}} \int_{\mathbb{B}} \frac{|f(z)-f(w)|^{p}\left(1-\left|\varphi_{a}(z)\right|^{2}\right)^{\frac{n+1}{2}}\left(1-\left|\varphi_{a}(w)\right|^{2}\right)^{\frac{n+1}{2}}}{\left|\varphi_{w}(z)\right|^{p}|1-\langle w, z\rangle|^{p}} d v_{t}(z) d v_{t}(w) \\
& \quad=\int_{\mathbb{B}} \int_{\mathbb{B}} \frac{\left|f \circ \varphi_{w}(u)-f \circ \varphi_{w}(0)\right|^{p}}{|u|^{p}}\left(1-\left|\varphi_{a}\left(\varphi_{w}(u)\right)\right|^{2}\right)^{\frac{n+1}{2}} d v_{t}(u)\left(1-\left|\varphi_{a}(w)\right|^{2}\right)^{\frac{n+1}{2}} d \lambda(w) \\
& \quad \leq C \int_{\mathbb{B}} \int_{\mathbb{B}}\left|\widetilde{\nabla} f \circ \varphi_{w}(u)\right|^{p}\left(1-\left|\varphi_{a}\left(\varphi_{w}(u)\right)\right|^{2}\right)^{\frac{n+1}{2}} d v_{t}(u)\left(1-\left|\varphi_{a}(w)\right|^{2}\right)^{\frac{n+1}{2}} d \lambda(w) \\
& \quad \leq C \int_{\mathbb{B}} \int_{\mathbb{B}}|\widetilde{\nabla} f(z)|^{p}\left(1-\left|\varphi_{w}(z)\right|^{2}\right)^{n+1+t}\left(1-\left|\varphi_{a}(z)\right|^{2}\right)^{\frac{n+1}{2}} d \lambda(z)\left(1-\left|\varphi_{a}(w)\right|^{2}\right)^{\frac{n+1}{2}} d \lambda(w) \\
& \quad \leq C K \int_{\mathbb{B}}|\widetilde{\nabla} f(z)|^{p}\left(1-\left|\varphi_{a}(z)\right|^{2}\right)^{n+1} d \lambda(z),
\end{aligned}
$$

where

$$
K=\sup _{a, z \in \mathbb{B}} \int_{\mathbb{B}} \frac{1}{\left(1-\left|\varphi_{a}(z)\right|^{2}\right)^{\frac{n+1}{2}}}\left(1-\left|\varphi_{w}(z)\right|^{2}\right)^{n+1+t}\left(1-\left|\varphi_{a}(w)\right|^{2}\right)^{\frac{n+1}{2}} d \lambda(w) .
$$

Employing the change of variables $w \mapsto \varphi_{z}(u)$ and using the fact that $\left|\varphi_{z}(w)\right|=\left|\varphi_{w}(z)\right|$ we have

$$
K=\sup _{a, z \in \mathbb{B}} \int_{\mathbb{B}} \frac{1}{\left(1-\left|\varphi_{z}(a)\right|^{2}\right)^{\frac{n+1}{2}}}\left(1-|u|^{2}\right)^{n+1+t}\left(1-\left|\varphi_{a}\left(\varphi_{z}(u)\right)\right|^{2}\right)^{\frac{n+1}{2}} d \lambda(u) .
$$

It follows from Lemma 2.5 and the fact that $\left|\left(\varphi_{a} \circ \varphi_{z}\right)(u)\right|=\left|\varphi_{\varphi_{z}(a)}(u)\right|$ (see [5]) that

$$
\begin{aligned}
K & =\sup _{a, z \in \mathbb{B}} \int_{\mathbb{B}} \frac{1}{\left(1-\left|\varphi_{z}(a)\right|^{2}\right)^{\frac{n+1}{2}}}\left(1-\left|\varphi_{\varphi_{z}(a)}(u)\right|^{2}\right)^{\frac{n+1}{2}} d v_{t}(u) \\
& =\sup _{a, z \in \mathbb{B}} \int_{\mathbb{B}} \frac{\left(1-|u|^{2}\right)^{\frac{n+1}{2}}}{\left|1-\left\langle u, \varphi_{z}(a)\right\rangle\right|^{n+1}} d v_{t}(u)=\sup _{w \in \mathbb{B}} \int_{\mathbb{B}} \frac{\left(1-|u|^{2}\right)^{\frac{n+1}{2}}}{|1-\langle u, w\rangle|^{n+1}} d v_{t}(u)<\infty .
\end{aligned}
$$

Combining (19) with (20), the result follows from Lemma 2.4.

By choosing $\alpha=0, \gamma=p / 2$, and $\beta=p / 2-(n+1)$ in Lemma 2.2 , similarly to the proof of Theorem 4.1 is obtained the following result.

Theorem 4.3 Assume that $f \in H(\mathbb{B})$ and $2(n+1)<p<\infty$. Then $f \in \mathcal{B}(\mathbb{B})$ if and only if

$$
\sup _{a \in \mathbb{B}} \int_{\mathbb{B}} \int_{\mathbb{B}}\left(\frac{|f(z)-f(w)|}{|1-\langle z, w\rangle|}\right)^{p}\left(1-\left|\varphi_{a}(w)\right|^{2}\right)^{n+1} d v_{t}(z) d v_{t}(w)<\infty
$$

where $t=(p-2(n+1)) / 2$. 
Using Theorem 4.3, similarly to the proof of Theorem 4.2 is proved the following result.

Theorem 4.4 Assume that $f \in H(\mathbb{B})$ and $2(n+1)<p<\infty$. Then $f \in \mathcal{B}(\mathbb{B})$ if and only if

$$
\sup _{a \in \mathbb{B}} \int_{\mathbb{B}} \int_{\mathbb{B}}\left(\frac{|f(z)-f(w)|}{\left|w-P_{w} z-s_{w} Q_{w} z\right|}\right)^{p}\left(1-\left|\varphi_{a}(w)\right|^{2}\right)^{n+1} d v_{t}(z) d v_{t}(w)<\infty
$$

where $t=(p-2(n+1)) / 2$

\section{Competing interests}

The authors declare that they have no competing interests.

Authors' contributions

All authors contributed equally to the writing of this paper. All authors read and approved the final manuscript.

\section{Author details}

${ }^{1}$ Department of Mathematics, JiaYing University, Meizhou, GuangDong 514015, China. ${ }^{2}$ Mathematical Institute of the Serbian Academy of Sciences, Knez Mihailova 36/III, Beograd, 11000, Serbia. ${ }^{3}$ Department of Mathematics, King Abdulaziz University, P.O. Box 80203, Jeddah, 21589, Saudi Arabia.

\section{Acknowledgements}

The first author of this paper is supported by the project of Department of Education of Guangdong Province (No. 2013KJCX0170) and NSF of Guangdong, China (S2013010011978).

Received: 23 March 2014 Accepted: 22 October 2014 Published: 19 Nov 2014

\section{References}

1. Zhu, K: Operator Theory on Function Spaces. Pure and Applied Mathematics, vol. 139. Dekker, New York (1990)

2. Hahn, K: Holomorphic mappings of the hyperbolic space into the complex Euclidean space and the Bloch theorem. Can. J. Math. 27, 446-458 (1975)

3. Timoney, R: Bloch functions in several complex variables I. Bull. Lond. Math. Soc. 12, 241-267 (1980)

4. Timoney, R: Bloch functions in several complex variables II. J. Reine Angew. Math. 319, 1-22 (1980)

5. Zhu, K: Spaces of Holomorphic Functions in the Unit Ball. Springer, New York (2005)

6. Zhang, M, Xu, W: Composition operators on $\alpha$-Bloch spaces of the unit ball. Acta Math. Sin. Engl. Ser. 23, 1991-2002 (2007)

7. Colonna, F: New criteria for boundedness and compactness of weighted composition operators mapping into the Bloch space. Cent. Eur. J. Math. 11(1), 55-73 (2013)

8. Krantz, S, Stević, S: On the iterated logarithmic Bloch space on the unit ball. Nonlinear Anal. TMA 71, 1772-1795 (2009)

9. Li, S, Stević, S: Composition followed by differentiation between Bloch type spaces. J. Comput. Anal. Appl. 9(2), 195-206 (2007)

10. Li, S, Stević, S: Integral type operators from mixed-norm spaces to $\alpha$-Bloch spaces. Integral Transforms Spec. Funct. 18(7), 485-493 (2007)

11. Li, S, Stević, S: Weighted composition operators from Bergman-type spaces into Bloch spaces. Proc. Indian Acad. Sci. Math. Sci. 117(3), 371-385 (2007)

12. Li, S, Stević, S: Weighted composition operators from $H^{\infty}$ to the Bloch space on the polydisc. Abstr. Appl. Anal. 2007, Article ID 48478 (2007)

13. Li, S, Stević, S: Generalized composition operators on Zygmund spaces and Bloch type spaces. J. Math. Anal. Appl. $338,1282-1295(2008)$

14. Li, S, Stević, S: Weighted composition operators from Zygmund spaces into Bloch spaces. Appl. Math. Comput. 206(2), 825-831 (2008)

15. Madigan, K, Matheson, A: A compact composition operators on the Bloch space. Trans. Am. Math. Soc. 347(7), 2679-2687 (1995)

16. Stević, S: Essential norms of weighted composition operators from the $\alpha$-Bloch space to a weighted-type space on the unit ball. Abstr. Appl. Anal. 2008, Article ID 279691 (2008)

17. Stević, S: Norms of some operators from Bergman spaces to weighted and Bloch-type space. Util. Math. 76, 59-64 (2008)

18. Stević, S: On a new operator from the logarithmic Bloch space to the Bloch-type space on the unit ball. Appl. Math. Comput. 206, 313-320 (2008)

19. Stević, S: Norm and essential norm of composition followed by differentiation from $\alpha$-Bloch spaces to $H_{\mu}^{\infty}$. Appl. Math. Comput. 207, 225-229 (2009)

20. Stević, S: On a new integral-type operator from the Bloch space to Bloch-type spaces on the unit ball. J. Math. Anal. Appl. 354, 426-434 (2009)

21. Stević, S: On an integral operator between Bloch-type spaces on the unit ball. Bull. Sci. Math. 134, $329-339$ (2010)

22. Nowak, M: Bloch and Möbius invariant Besov spaces on the unit ball of $\mathbb{C}^{n}$. Complex Var. Theory Appl. 44, 1-12 (2001) 
23. Ren, G, Tu, C: Bloch space in the unit ball of $\mathbb{C}^{n}$. Proc. Am. Math. Soc. 133, 719-726 (2005)

24. Li, S, Wulan, H: Characterizations of $\alpha$-Bloch spaces on the unit ball. J. Math. Anal. Appl. 343, 58-63 (2008)

25. Stević, S: Boundedness and compactness of an integral operator on a weighted space on the polydisc. Indian J. Pure Appl. Math. 37, 343-355 (2006)

26. Stević, S: On Lipschitz and $\alpha$-Bloch spaces on the unit polydisc. Studia Sci. Math. Hung. 45, 361-378 (2008)

27. Avetisyan, K: Hardy-Bloch type spaces and lacunary series on the polydisk. Glasg. Math. J. 49, 345-356 (2007)

28. Avetisyan, K: Weighted integrals and Bloch spaces of $n$-harmonic functions on the polydisc. Potential Anal. 29, 49-63 (2008)

29. Clahane, D, Stević, S: Norm equivalence and composition operators between Bloch/Lipschitz spaces of the unit ball. J. Inequal. Appl. 2006, Article ID 61018 (2006)

30. Li, B, Ouyang, C: Higher radial derivative of Bloch type functions. Acta Math. Sci. 22, 433-445 (2002)

31. Li, S, Stević, S: Some characterizations of the Besov space and the $\alpha$-Bloch space. J. Math. Anal. Appl. 346, 262-273 (2008)

32. Li, S, Wulan, H: Some new characterizations of Bloch spaces. Taiwan. J. Math. 14(6), 2245-2259 (2010)

33. Meng, X: Some results on $Q_{K}(p, q)$ space. Abstr. Appl. Anal. 2008, Article ID 404636 (2008)

34. Nowak, M: Bloch space on the unit ball of $\mathbb{C}^{n}$. Ann. Acad. Sci. Fenn., Math. 23, 461-473 (1998)

35. Ouyang, C, Yang, W, Zhao, R: Characterizations of Bergman spaces and the Bloch space in the unit ball of $\mathbb{C}^{n}$. Trans. Am. Math. Soc. 374, 4301-4312 (1995)

36. Ouyang, C, Yang, W, Zhao, R: Móbius invariant $Q_{p}$ spaces associated with the Green's function on the unit ball of $\mathbb{C}^{n}$. Pac. J. Math. 182, 69-99 (1998)

37. Stević, S: On Bloch-type functions with Hadamard gaps. Abstr. Appl. Anal. 2007, Article ID 39176 (2007)

38. Stević, S: Bloch-type functions with Hadamard gaps. Appl. Math. Comput. 208, 416-422 (2009)

39. Stević, S: Harmonic Bloch and Besov spaces on the unit ball. Ars Comb. 91, 3-9 (2009)

40. Wulan, H, Zhu, K: Derivative-free characterizations of $Q_{K}$ spaces. J. Aust. Math. Soc. 82, 283-295 (2007)

41. Wulan, H, Zhu, K: Lacunary series in $Q_{K}$ spaces. Stud. Math. 178, 217-230 (2007)

42. Wulan, H, Zhu, K: Bloch and BMO functions in the unit ball. Complex Var. Theory Appl. 53, 1009-1019 (2008)

43. Wulan, $H$, Zhu, K: $Q_{K}$ spaces via higher order derivatives. Rocky Mt. J. Math. 38, 329-350 (2008)

44. Yang, W: Some characterizations of $\alpha$-Bloch spaces on the unit ball of $\mathbb{C}^{n}$. Acta Math. Sci. 17, 471-477 (1997)

45. Zhao, R: On $\alpha$-Bloch functions and VMOA. Acta Math. Sci. 16, 349-360 (1996)

46. Zhao, R: On a general family of function spaces. Ann. Acad. Sci. Fenn., Math. Diss. 105, 56 pp. (1996)

47. Zhao, R: A characterization of Bloch-type spaces on the unit ball of $\mathbb{C}^{n}$. J. Math. Anal. Appl. 330, 291-297 (2007)

48. Zhou, J: Lacunary series in $Q_{K}$ type spaces. J. Funct. Spaces Appl. 6, 293-301 (2008)

49. Zhu, K: Bloch type spaces of analytic functions. Rocky Mt. J. Math. 23, 1143-1177 (1993)

50. Rudin, W: Function Theory in the Unit Ball of $\mathbb{C}^{n}$. Springer, New York (1980)

51. Li, S, Wulan, H, Zhao, R, Zhu, K: A characterization of Bergman spaces on the unit ball of $\mathbb{C}^{n}$. Glasg. Math. J. 51 315-330 (2009)

\section{Submit your manuscript to a SpringerOpen ${ }^{\ominus}$ journal and benefit from:}

- Convenient online submission

Rigorous peer review

- Immediate publication on acceptance

- Open access: articles freely available online

- High visibility within the field

- Retaining the copyright to your article 